\title{
Mathematical models of sow reproduction
}

\author{
W. H. Close and J. E. Pettigrew* \\ AFRC Institute for Grassland and Animal Production, Shinfield, Reading RG2 9AQ,UK; and \\ * Department of Animal Science, University of Minnesota, MN 55108, USA
}

Keywords: pig; models; metabolism

Reproductive failure is the major reason for premature culling of sows (Dagorn \& Aumaitre, 1979; Kroes \& Van Male, 1979), which in turn reduces overall reproductive efficiency. A major component of this failure is delayed resumption of oestrous activity after weaning (Reese et al., 1982; King \& Williams, 1984; Mullan \& Williams, 1989). The losses of both body fat and lean during lactation (Britt et al., 1988; Mullan \& Close, 1989) have been implicated in the interruption of reproductive function after weaning, but it is not clear what metabolic and endocrine signals connect tissue loss with reproductive function. It is likely that both the levels of body reserves, relative to some threshold level, and concentrations of metabolites and metabolic hormones are related to reproduction, but these depend upon the nutrient intake of the animal. It follows, therefore, that any procedure which predicts the effects of nutrient intake on these phenomena will facilitate our attempts to design feeding strategies to ensure optimal reproductive efficiency.

The objective of this paper is to discuss recent developments in mathematical modelling of the response of sows to their diet, and how the predictions of these models may be used to anticipate production responses which may ultimately lead to an improvement in sow performance.

\section{Types of models}

There are basically two kinds of models; empirical and mechanistic. An empirical model describes the response of an animal to a given set of circumstances and usually attempts to develop prediction equations from experimental data sets using biometric procedures. Its usefulness is limited to the experimental conditions under which it was developed and it should not be extended beyond the range of conditions under which the original observations were made. Such models usually operate at the 'whole animal' level and are not dynamic. Empirical models will not be considered further in this paper.

Mechanistic animal models, on the other hand, deal with the effects of diet and other external variables on processes within the animal and use these to predict whole-animal responses. Thus, depending upon the knowledge available and the level of sophistication, the model may operate at the tissue, cellular or molecular level. Because they operate at a lower level of organization, these approaches are more flexible and may be expected to predict responses over a wide range of conditions. This paper describes two such mechanistic models which operate at different levels of sophistication, and which may be used to predict animal responses to a series of nutritional and managemental inputs.

\section{A factorial model of the pregnant and lactating sow}

This model (the nutrient partitioning model) operates on the factorial principle that dietary nutrients may be partitioned between the requirements to maintain the animal and to deposit tissue 
(protein and fat) either in the maternal body or products formed but subsequently lost from the body. For the pregnant animal the initial step is to calculate the rate of protein deposition, and hence lean tissue growth rate, in relation to daily feed intake for animals of different body weights. The 'linear/plateau' or 'bent stick' approach has been adopted in the current model because at relatively low feed intakes protein deposition is limited by the daily supply of protein and energy and growth rate therefore responds linearly to feed intake.. As feed intake increases, a point is reached at which protein deposition is at a maximum, depending upon the genetic potential of the animal and its stage of development, and beyond which there is no further increase in protein deposition with increased feed intake. Such an approach has been previously described for pigs and poultry (ARC, 1981; Morris, 1983).

Once the relationship between feed intake and protein deposition has been described, the response of the pregnant sow to increasing levels of feeding may be determined. The first priority is for maintenance, followed by the requirement for protein deposition and hence lean tissue growth. Once these requirements have been met, any additional nutrients are channelled into fat deposition. However, when nutrient intake is below the requirement for both maintenance and protein deposition, as may occur in late pregnancy, then mobilization of fat occurs. The model is iterative and, since it includes equations to predict the growth of the products of conception (Noblet et al., 1985), both total and net body weight gain of the sow can be calculated throughout pregnancy.

During lactation the primary nutrient requirements are for maintenance and milk production, and any additional nutrients will be available for deposition of protein and fat in the maternal body. The nutrient requirement for milk production is based on the rate of tissue accretion in suckling piglets of different body weights, litter sizes and stages of lactation. If the requirements for maintenance and milk are above the sow's voluntary feed intake, then body tissue is mobilized and the appropriate change in lean and fat reserves, and hence body weight, can be predicted. Thus, for any given nutritional, environmental and husbandry situation, changes in body weight and in lean and fat reserves during both pregnancy and lactation may be calculated. A brief summary of the principal components of this factorial model is presented in Table 1. More detailed information is provided by Williams et al. (1985) and Mullan et al. (1989).

Table 1. The principal components of a factorial model to predict the response of the sow during pregnancy and lactation

(1) Determination of the relation between nitrogen intake and nitrogen retention

(2) Calculation of the energy and nitrogen requirements for maintenance

(3) The nutrient requirements for conceptus growth during pregnancy

(4) Estimation of the nutrient requirements for milk production based on the rates of tissue accretion in suckling piglets

(5) Calculation of the net gain or loss of protein and fat in the maternal body and hence changes in body weight and in backfat thickness

(6) Estimation of the voluntary feed intake of the sow during lactation

(7) Assessment of the environmental cost associated with poor housing 


\section{A.model of lactating sow metabolism}

The connection between diet and reproduction may be assumed to involve dietary effects on voluntary food intake, digestion and absorption of nutrients, metabolism of absorbed nutrients and endocrine function. Thus mathematical modelling has been used to attempt to trace these dietary effects both systematically and quantitatively. The present metabolic model involves only that part of the connection between diet and reproduction that deals with the metabolism of absorbed nutrients. The model is structured to work throughout the reproductive cycle, but to date the inputs have been developed only for lactation.

The structure of the model is shown in Fig. 1. The model is driven by quantities of absorbed nutrients (DLy, DAa, etc.). It monitors the sizes of several pools (boxes) of metabolites, body stores and milk components. It is dynamic, that is, it moves through time, calculating at each time step the rate of movement of material along each arrow in Fig. 1 and solving a differential equation for each pool to determine the current size of the pool and thus metabolite concentrations.

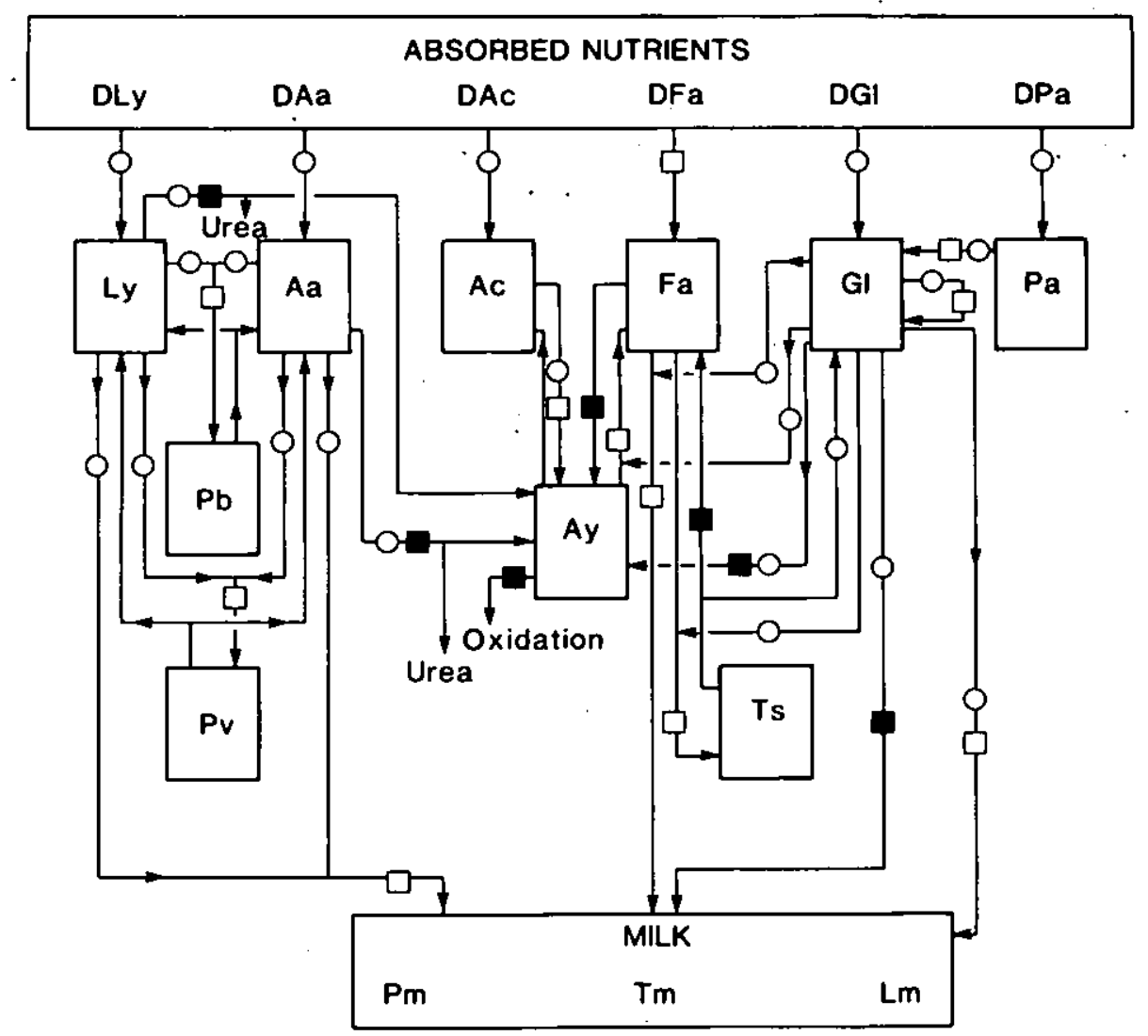

Fig. 1. Schematic representation of model. The model is driven by continuous inputs of absorbed nutrients (DLy, etc.). Pools specifically represented include lysine (Ly), other amino acids $(\mathrm{Aa})$, acetic acid $(\mathrm{Ac})$, fatty acids $(\mathrm{Fa})$, glucose $(\mathrm{Gl})$, propionic acid $(\mathrm{Pa})$, acetyl-Co $\mathrm{A}$ $(\mathrm{Ay})$, body protein $(\mathrm{Pb})$, visceral protein $(\mathrm{Pv})$, storage triacylglycerol $(\mathrm{Ts})$ and protein $(\mathrm{Pm})$, fat $(\mathrm{Tm})$ and lactose $(\mathrm{Lm})$ in milk. Fluxes requiring/yielding ATP indicated by $O$ (uses ATP in transport), $\square$ (uses ATP in reaction) and $\square$ (produces ATP in reaction). 
A key aspect of this type of model is the estimation of the rates of flow of materials among pools according to 3 kinetic principles. The first is that the rate of each transaction is a function of the physiological conditions within the animal, especially metabolite concentrations. The second is that the transactions are saturable with substrate and that the rates can be approximated by mathematical formulae often applied to saturable systems, as described below. The third principle is that the rate is expressed per unit tissue mass, allowing scaling for body size.

The mathematical treatment of the kinetics of saturable systems was outlined by Pettigrew et al. (1989a). It is assumed that many of the control systems of the body produce responses that approximate to the following general equation for the rate of utilization of a principal substrate and which is widely used in biology:

$$
\text { Rate }=\mathrm{V}_{\max } /\left(\mathrm{t}+\left(\mathrm{K} /[\mathrm{S}]^{\mathrm{E}}\right)\right.
$$

where $\mathrm{V}_{\max }$ is the maximum velocity per unit of tissue; $\mathrm{K}$ is the affinity constant for the specific substrate; $[S]$ is the concentration of the substrate; and $E$ is the steepness parameter. Inhibition constants $(\mathrm{J})$ can also be included in the general equation.

The value of $E$ is usually 1 . At low substrate concentration, the rate is higher when the value of the affinity constant (K) is lower. Physiological priorities for one transaction over another can be simulated in the model by assigning a lower value of $\mathrm{K}$ to the higher-priority transaction. Inhibitors may be simulated and multiple terms may be included in a rate equation to reflect multiple substrates and/or inhibitors.

The parameters of the rate equations $\left(\mathrm{V}_{\max }, \mathrm{K}, \mathrm{E}\right.$ and $\left.\mathrm{J}\right)$ must be part of the model. There are limited data from which many of these values can be estimated directly, and so they have been estimated indirectly in various ways from data in the literature. They are therefore consistent with those data and with common concepts of physiology, biochemistry and metabolism.

The entire model was tested by simulating a total of 16 experimental treatments reported in 4 publications. The results of the simulations were compared with experimental data reported in the publications and suggest that the performance of the model is encouraging but imperfect (Pettigrew et al., 1989b, c). Testing continues.

Plans for further development of the model involve current animal studies to provide the necessary information to extend and validate it. In addition it is planned to include the incorporation of metabolic hormones, direct estimation of kinetic parameters and extension of the model to cover pregnancy and the post-weaning period. The connections between model pools (metabolites, metabolic hormones, body stores) and reproduction should eventually be explored.

\section{Uses of models}

The ultimate aim of these models is to improve our understanding of factors influencing the efficiency of reproduction in sows. They contribute to this goal in several ways.

\section{Design and evaluation of feeding programmes}

This is the primary objective of the nutrient partitioning model and a secondary objective of the metabolic model. Proposed feeding programmes can be evaluated quickly and inexpensively by the use of a model (Fig. 2) and appropriate feeding programmes can be identified and compared. Components of the feeding programme which can be evaluated include the energy and protein (amino acid) supply during various phases of the reproductive cycle.

\section{Understanding the biological system}

This is the primary objective of the metabolic model and a secondary objective of the nutrient partitioning model. 


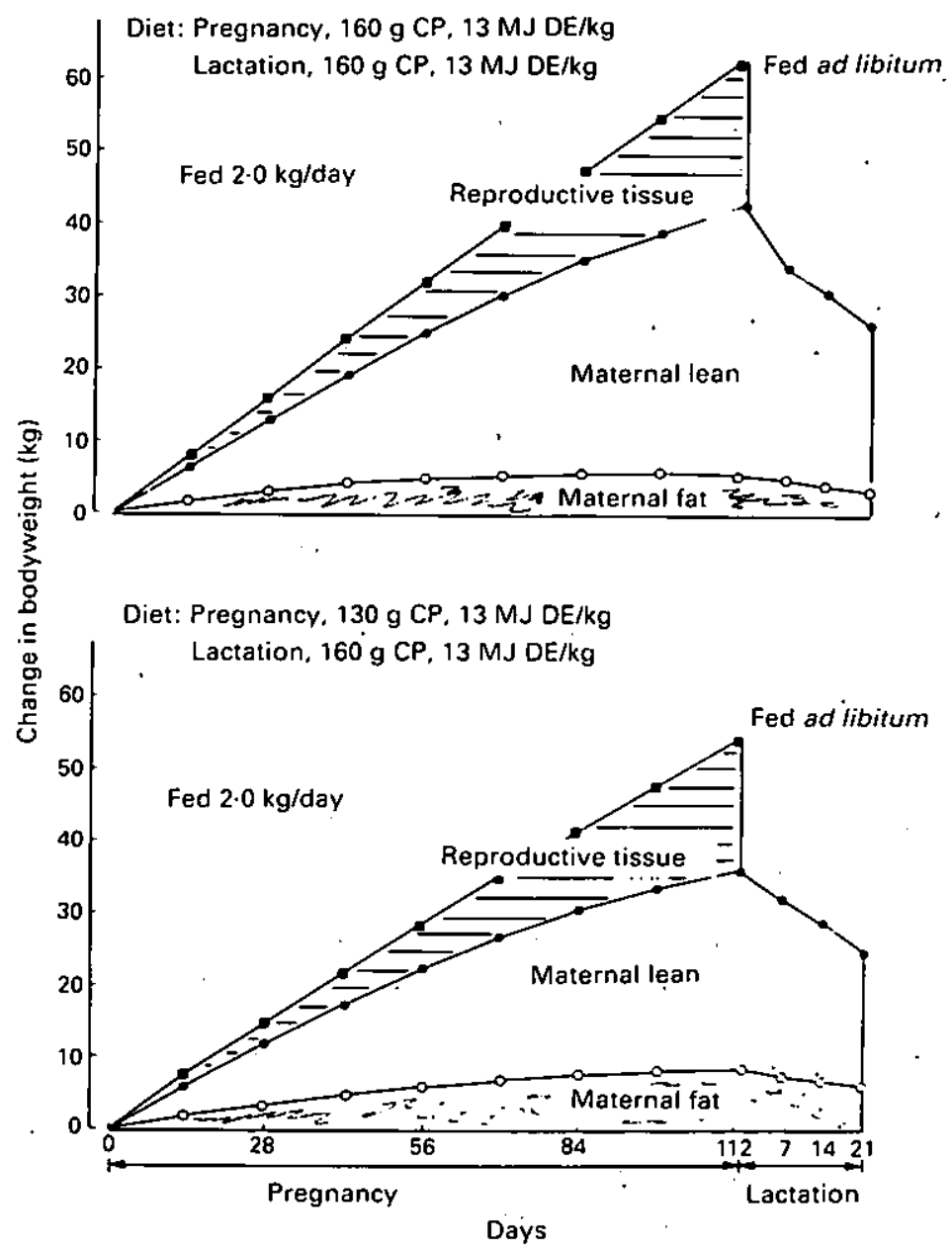

Fig. 2. Model predictions showing the effects of different feeding regimens during pregnancy on the change in body weight and in lean and fat deposition in young gilts $(120 \mathrm{~kg}$ body weight at mating) during their first parity.

Development of a model necessitates a logical, systematic and quantitative consideration of the biological system which may sometimes lead to insights unlikely to be obtained without the rigorous and quantitative processes of modelling. Similarly, use of the completed models may lead to new concepts and hypotheses.

Mathematical modelling requires many different data sets dealing with different subjects and can thus identify important areas in which information is inadequate or nonexistent. It also helps to clarify questions to be addressed by animal research.

Experience with the use of mathematical models will allow preliminary evaluation of 'What if?' scenarios. Results may direct our thinking into novel areas and may lead to hypotheses concerning the effects of various feeding strategies on animal performance. These strategies should then be evaluated by animal experimentation.

\section{Research efficiency}

Resources available for biological research will continue to be limited. It is therefore imperative that these resources be used as efficiently as possible. Extensive feeding trials over multiple parities 
require a long time interval and considerable resources, and so such experiments must address the most important questions and evaluate key strategies. As discussed above, a model can provide preliminary evaluation of feeding programmes so that the most promising can be selected for evaluation by feeding trial. Models can also direct animal studies dealing with physiological mechanisms to the key questions. Similarly, the contribution which any given data set can make to our understanding of sow biology and production can be enhanced by including it in a mathematical model with many other data sets. The entire body of data organized into a mathematical mode! contributes more than the sum of its parts.

\section{Summary and conclusions}

Nutrition affects reproduction, but the physiological mechanisms are not known. Defining those mechanisms is a high priority for animal scientists. This paper briefly describes mathematical models developed to aid in elucidating those mechanisms and which may be applied to predict animal performance.

Two types of mechanistic mathematical models of sows are described, based respectively on nutrient partitioning and on metabolic and physiological principles. The nutrient partitioning model is relatively mature but the metabolic/physiological model is still at an early stage of development. The use of such models in the design and evaluation of feeding programmes, in understanding the biological system and in improving research efficiency are outlined.

These two models are now being used as described, and it is anticipated that they, and other models, will make important contributions to the marked improvements in reproductive performance in commercial pig production that is anticipated during the next few years.

\section{References}

Agricultural Research Council (1981) The Nutrient Requirements of Pigs. C.A.B., Slough.

Britt, J.H., Armstrong, J.D. \& Cox, N.M. (1988) Metabolic interfaces between nutrition and reproduction in pigs. Proc. $/ \mathrm{lth} \mathrm{Im}$. Congr. Anim. Reprod. \& A.I., Dublin, pp. $117-125$.

Dagorn, J. \& Aumaitre, A. (1979) Sow culling: reasons for an effect on productivity. Livestock Prod. Sci. 6, $167-177$.

King, R.H. \& Williams, I.H. (1984) The effect of nutrition on the reproductive performance of first-litter sows. 1 . Feeding level during lactation and between weaning and mating. Anim. Prod. 38, 241-247.

Kroes, Y. \& van Male, J.P. (1979) Reproductive lifetime of sows in relation to economy of production. Livestock Prod. Sci. 6, 179-183.

Morris, T.R. (1983) The interpretation of response data from animal feeding trials. In Recen Advances in Animal Nutrition-1983, pp. 13-23. Ed. W. Haresign. Butterworth, London.

Mullan, B.P. \& Close, W.H., (1989) The partition and utilisation of energy and nitrogen by sows during their first lactation. Anim. Prod. 48, 627-637.

Mullan, B.P. \& Williams, I.H. (1989) The effects of body reserves at farrowing on the reproductive performance of first-litter sows. Anim. Prod. 48, 449-457.

Mullan, B.P., Close, W.H. \& Cole, D.J.A. (1989) Predicting nutrient responses of the lactating sow. In Recent Advances in Animal Nutrition-1989, pp. 229-243. Ed. W. Haresign. Butterworth, London.
Noblet, J., Close, W.H., Heavens, R.P. \& Brown, D. (1985) Studies on the energy metabolism of the pregnant sow. I. Uterus and mammary tissue development. Br. J. Nutr. 53, 251-265.

Pettigrew, J.E., Gill, M., France, J. \& Close, W.H. (1989a) A mathematical model of sow energy and protein metabolism. In Energy Metabolism of Farm Animals, pp. 199-202. Eds Y. van der Honing \& W. H. Close. EAAP Publication No. 43, Pudoc, Wageningen.

Pettigrew, J.E., Gill, M., France, J. \& Close, W.H. (1989b) Connecting the sow diet to reproductive performance: the protein section of a mathematical model of sow metabolism. J. Anim. Sci. 67, Suppl. 2, 120.

Pettigrew, J.E., Gill, M., France, J. \& Close, W.H. (1989c) The intermediary and fat metabolism parts of a mathematical model of sow metabolism. J. Anim. Sci. 67, Suppl. 1, 257.

Reese, D.E., Moser, B.D., Peo, E.R., Jr, Lewis, A.J., Zimmerman, D.R., Kinder, J.E. \& Stroup, W.W. (1982) lnfluence of energy intake during lactation on the interval between weaning to first estrus in sows. $J$. Anim. Sci. 55, 590-598.

Williams, I.H., Close, W.H. \& Cole, D.J.A. (1985) Strategies for sow nutrition: predicting the response of pregnant animals to protein and energy intake. In Recemt Advances in Animal Nutrition-1985, pp. 133-147. Eds W. Haresign \& D. J. A. Cole. Butterworth, London. 